\title{
Relative abdominal adiposity is associated with chronic low back pain: a preliminary explorative study
}

\author{
Cristy Brooks ${ }^{*} \mathbb{D}$, Jason C. Siegler ${ }^{2}$ and Paul W. M. Marshall ${ }^{2}$
}

\begin{abstract}
Background: Although previous research suggests a relationship between chronic low back pain (CLBP) and adiposity, this relationship is poorly understood. No research has explored the relationship between abdominalspecific subcutaneous and visceral adiposity with pain and disability in CLBP individuals. The aim of this study therefore was to examine the relationship of regional and total body adiposity to pain and disability in CLBP individuals.

Methods: A preliminary explorative study design of seventy $(n=70)$ adult men and women with CLBP was employed. Anthropometric and adiposity measures were collected, including body mass index, waist-to-hip ratio, total body adiposity and specific ultrasound-based abdominal adiposity measurements. Self-reported pain and disability were measured using a Visual Analogue Scale (VAS) and the Oswestry Disability Index (ODI) questionnaires respectively. Relationships between anthropometric and adiposity measures with pain and disability were assessed using correlation and regression analyses.
\end{abstract}

Results: Significant correlations between abdominal to lumbar adiposity ratio (A-L) variables and the waist-to-hip ratio with self-reported pain were observed. A-L variables were found to predict pain, with $9.1-30.5 \%$ of the variance in pain across the three analysis models explained by these variables. No relationships between anthropometric or adiposity variables to self-reported disability were identified.

Conclusions: The findings of this study indicated that regional distribution of adiposity via the A-L is associated with $C L B P$, providing a rationale for future research on adiposity and $C L B P$.

Keywords: Chronic low back pain, Obesity, Abdominal adiposity, Ultrasound, Pain, Disability

\section{Background}

cLBP places a large economic burden on society, with loss of income and treatment costs in Australia in excess of $\$ 9$ billion annually [1]. Low back pain (LBP) affects $10 \%$ of the global population and is ranked as the 7th leading disability in the world and the highest ranked for years lived with the disability [2]. Obesity is also a costly and prevalent health condition, which has been previously linked to cLBP [3-13]. In the past this relationship has been demonstrated using body mass index (BMI) as a measure of obesity $[3,6,8,10,14]$, which has been

\footnotetext{
* Correspondence: c.brooks@westernsydney.edu.au

${ }^{1}$ School of Science and Health, Western Sydney University, Locked Bag

1797Campbelltown Campus, Sydney, NSW 2751, Australia

Full list of author information is available at the end of the article
}

defined as an individual's body weight divided by their height squared [15]. Despite its common use, the simplicity of BMI and its disregard for body composition [12] have led to its criticism and greater emphasis on alternative obesity measurements. This shift in focus is important because research suggests that adipose tissue may be of consequence in the pathogenesis of chronic pain conditions [12]. For example, increased adiposity (total body, upper and lower limbs, trunk, android and gynoid) is associated with higher levels of LBP intensity and disability [12]. Ultrasound (US) may be a suitable substitute for BMI and other simplistic obesity measurements as it is a valid and reliable measurement tool of assessing adiposity when compared to gold standard 
methods [16-21]. However, US has not yet been utilized in CLBP research.

Although there is an established relationship between adiposity and low back pain [12], the inconsistent and poorly defined terminology used in the past makes previous research confusing and difficult to draw conclusions from. Moreover, there is a lack of research on the distribution of adiposity and its possible relationship with pain and disability levels in cLBP. No studies have investigated whether regionally accumulated abdominal adiposity may be of more relevance than total body adiposity in a cLBP population. For example, visceral adiposity has been suggested to be more important than total adiposity in the risk of developing obesity-related disorders [20, 22]. Visceral adiposity has also been suggested to be of greater consequence to the metabolic profile $[16,23]$ and various medical pathologies [24] than subcutaneous adipose tissue, on the basis of physiological and metabolic differences such as adipocyte size and lipolytic activity [25]. It may then be suggested that the distribution of excess visceral adipose tissue could also be associated with increased pain in cLBP individuals. Several plausible mechanisms for a cLBP-visceral adiposity relationship exist, including inflammatory processes occurring from adipose tissue or increased mechanical load on the lumbar spine and surrounding structures produced by excess adiposity [12]. However, the cLBP-obesity relationship remains largely unknown, since research on the relationship between adiposity, primarily visceral, and cLBP is lacking.

In the exploration of the relative importance of regional versus total body adiposity, it is reasonable to believe that greater accumulation of adipose tissue in the abdominal region when compared to the lumbar region may also be of significance in the relationship to pain and disability in cLBP. This abdominal to lumbar adiposity ratio may be important, as greater abdominal adiposity could have flow-on effects for cLBP sufferers beyond that of an increase in body weight. For example, increased abdominal adipose tissue may result in the adoption of a compensatory hyperlordotic posture to counteract the constant anterior flexion torque placed on the lumbar spine. This excess anterior mass is worthy of investigation, since an increase in compressive force may predispose the spine to injury [26]. Irrespective of the potential metabolic or biomechanical mechanisms that may be responsible for such a relationship, the parameters of a possible association between adiposity and cLBP should first be examined.

As a result of the inconsistencies of previous research and the potentially important consequences of visceral adiposity on the persistence of cLBP via metabolic factors such as the stimulation of inflammatory processes, it is warranted to examine the significance of adiposity distribution and particularly visceral adiposity on the obesity-cLBP relationship. US may then be employed to investigate the possible importance of visceral adipose tissue, since it has been shown to be a valid and reliable method of assessing abdominal adiposity [16-21]. Therefore, the aim of this study was to examine the relationship between regional and total body adiposity with pain and disability in cLBP individuals. The experimental objectives of this study were: 1 ) To use US-derived ratios to assess abdominal adipose tissue distribution in individuals with cLBP, 2) To perform correlation and regression analyses to examine relationships between anthropometric and adiposity variables with self-reported pain and disability in cLBP individuals, and 3) To perform the correlation and regression analyses on pain and disability subgroups within the cLBP dataset. The hypothesis of this study was that greater abdominal adiposity, particularly visceral, would be associated with increased self-reported pain and disability in a cLBP population.

\section{Methods \\ Study design}

A preliminary explorative study design was employed to examine the relationship between adiposity distribution with pain and disability in a cLBP population. All participant data was collected at a tertiary education facility in Western Sydney, Australia, over a three-year period with two cycles of participant recruitment and data collection.

\section{Study population}

Seventy $(n=70)$ adult men and women aged $18-76$ years were included in the study and were recruited through the use of media advertising and leaflet drops in the local area. All included participants had cLBP (pain between the costal margin and gluteal fold for a minimum of three months). Participants were excluded if they had a history of spinal surgery, spinal fracture, diagnosed lumbar disc herniation (and attained a positive result on the straight leg raise test), existing bone, cardiac or nervous system condition, diagnosed severe mental illness, severe postural abnormality, pain radiating below the knee or diagnosed inflammatory joint disease. Written informed consent was provided by all participants. This study had ethical approval for research on human subjects by the Human Research Ethics Committee review board on the basis of the Declaration of Helsinki.

\section{Anthropometric measures}

Height, weight, waist circumference (WC), hip circumference, BMI and waist-to-hip ratio (WHR) were measured while participants were barefoot and wearing lightweight clothing. Height was measured using a wallmounted stadiometer (Veeder-Root high speed counter, 
Elizabethtown, N.C.) and recorded to the nearest $0.1 \mathrm{~cm}$. Weight was measured using a calibrated digital scale (A\&D UC-321, A\&D Co., Ltd) and recorded to the nearest $0.1 \mathrm{~kg}$. Waist circumference was measured using an anthropometric tape measure (Lufkin Executive Diameter Pocket Tape W606PM) at the narrowest point between the costal margin and the iliac crest and recorded to the nearest $0.1 \mathrm{~cm}$. Hip circumference was measured at the widest point of buttocks approximately level with the greater trochanters of the femur and recorded to the nearest $0.1 \mathrm{~cm}$. BMI was calculated as weight divided by height squared $\left(\mathrm{kg} / \mathrm{m}^{2}\right)$ [15]. WHR was calculated as WC divided by hip circumference.

\section{Adiposity measures}

\section{Total body adiposity}

Total body adiposity was measured using bioelectrical impedance analysis (BIA) (Metagenics VLA50, variation of ImpDF50, ImpediMed Limited, Eight Mile Plains, QLD, 2005), which has been shown to be a valid and reliable method when compared to gold standard methods [27-34]. Participants were required to refrain from food, drink and exercise 2 hours prior to the test and avoid alcohol in the 12 hours prior. Immediately prior to the test, participants emptied their bladder and lay supine on a plinth for 5 minutes to stabilise body fluids. The participant remained in this position with arms by their sides for the duration of the test. Pairs of electrodes $(\mathrm{Ag} /$ $\mathrm{AgCl} 3 \mathrm{~cm}$ diameter, Kendall Medi-Trace 100, Tyco Healthcare Group LP, Mansfield, MA) were placed on their hand and foot on the right side of the body. Prior to electrode placement, the skin was adequately prepared using a safety razor, fine abrasion tape and alcohol swabs to remove excess hair and reduce impedance. The hand electrodes were placed between the radial and ulna styloid processes $1 \mathrm{~cm}$ proximal to the metacarpophalangeal joint of the middle finger. The foot electrodes were placed between the medial and lateral malleoli of the tibia and fibula, respectively, and $1 \mathrm{~cm}$ proximal to the metatarsophalangeal joint of the middle toe. Each electrode pair was a minimum of $10 \mathrm{~cm}$ apart. Resistance and reactance was recorded from the BIA device and then used to calculate total body adiposity percentage from the BIA software.

\section{Regional adiposity}

Regional adiposity (including lumbar, supra-iliac and multiple abdominal sites) was measured with US using previously validated and reliable methods [16]. Five (5) subcutaneous adiposity and two (2) visceral adiposity measurements were conducted over five (5) anatomical locations on the surface of the skin in the trunk region of each participant, of which five (5) have been described elsewhere [16]. Details and images of each measurement are listed in Table 1 and shown in Figs. 1 and 2 respectively. Participants were required to lie supine for a period of 10 minutes prior to US testing to allow body fluids to stabilise. Each measurement required the use of conductive gel to gain a clear image.

\section{Adiposity ratios}

The adiposity ratios calculated from ultrasound-derived adiposity thickness measurements are defined in Table 2, of which one has been previously described [16]. Such ratios were worthy of inclusion as past research has questioned simplistic anthropometric measurements such as BMI and WHR due to their lack of sensitivity and specificity $[12,35,36]$. Additionally, existing evidence implies a relationship between adiposity and pain that may be complex and multifactorial [12]. Consequently, the examination of adiposity relative to the

Table 1 Ultrasound measurements

\begin{tabular}{|c|c|c|c|}
\hline Measurement & Probe & Anatomical location & Method used for measurement \\
\hline $\mathrm{msA}$ & Linear & Just below the xiphoid process of the sternum & $\begin{array}{l}\text { Minimum distance between the fat-skin barrier and the } \\
\text { anterior surface of the linea alba }\end{array}$ \\
\hline MppA & Linear & $\begin{array}{l}\text { Just below the surface of the xiphoid process of the } \\
\text { sternum (same anatomical position as the minimum } \\
\text { subcutaneous adiposity measurement) }\end{array}$ & $\begin{array}{l}\text { Maximum distance between the posterior surface of the } \\
\text { linea alba and the anterior surface of the peritoneum } \\
\text { covering the liver }\end{array}$ \\
\hline MsA & Linear & $\begin{array}{l}\text { (A) } 2 \mathrm{~cm} \text { above the umbilicus and (B) } 2 \mathrm{~cm} \text { below } \\
\text { the umbilicus }\end{array}$ & $\begin{array}{l}\text { Maximum distance between the fat-skin barrier and } \\
\text { the anterior surface of the linea alba }\end{array}$ \\
\hline MiA & Convex & $\begin{array}{l}2 \mathrm{~cm} \text { above the umbilicus (same anatomical position } \\
\text { as maximum subcutaneous abdominal adiposity } \mathrm{A} \text { ) }\end{array}$ & $\begin{array}{l}\text { Maximum distance between the posterior surface of } \\
\text { the rectus abdominis muscle and the anterior wall of } \\
\text { the abdominal aorta }\end{array}$ \\
\hline $\mathrm{MsSI}$ & Linear & Just above the iliac crest on the mid-axillary line & $\begin{array}{l}\text { Maximum distance between the fat-skin barrier and the } \\
\text { anterior surface of the external oblique muscle }\end{array}$ \\
\hline MsL & Linear & $\begin{array}{l}\text { Level of } L 4 / L 5 \text { directly over the lumbar erector } \\
\text { spinae muscle }\end{array}$ & $\begin{array}{l}\text { Maximum distance between the fat-skin barrier and the } \\
\text { anterior surface of the lumbar erector spinae muscle }\end{array}$ \\
\hline
\end{tabular}

MsA minimum subcutaneous abdominal adiposity, MppA maximum pre-peritoneal abdominal adiposity, MsA maximum subcutaneous abdominal adiposity, MiA maximum intra-abdominal adiposity, MsSI maximum subcutaneous supra-iliac adiposity, MsL maximum subcutaneous lumbar adiposity 


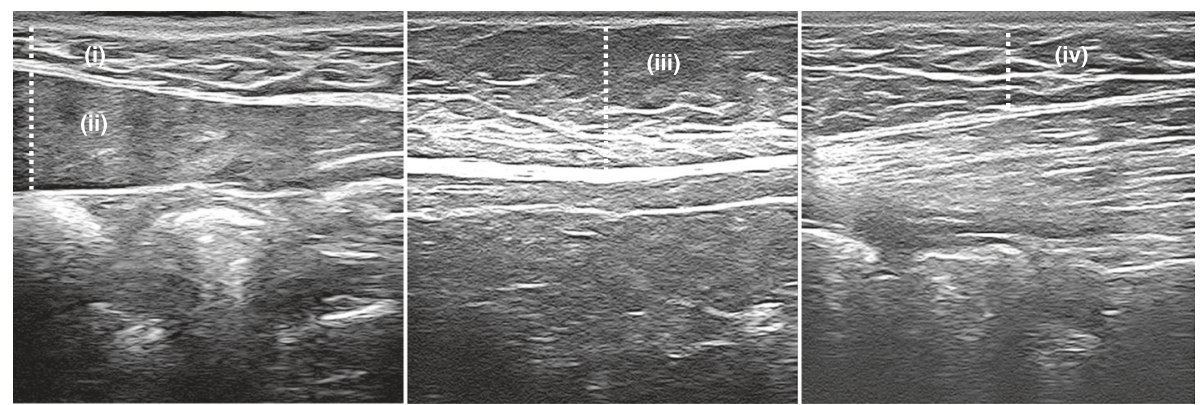

Fig. 1 Examples of abdominal US measurements (i) minimum subcutaneous abdominal adiposity (ii) maximum pre-peritoneal abdominal adiposity (iii) maximum subcutaneous abdominal adiposity A (iv) maximum subcutaneous abdominal adiposity $B$

individual may be crucial to better understanding the relationship between adiposity and cLBP.

\section{Pain and disability}

Self-reported pain was measured using a VAS, with 'no pain' on the left anchor and 'worst pain imaginable' on the right anchor, whereby the participant rated their current CLBP on a $100 \mathrm{~mm}$ line [37, 38]. Self-reported disability was measured using the ODI questionnaire, whereby participants filled in a 10-item questionnaire that was scored and converted to a percentage [37-39]. VAS and ODI have been previously shown to be valid and reliable methods of measuring self-reported pain and disability respectively in pain research, including cLBP populations [40-45].

\section{Statistical analysis}

Statistical analyses were performed using SPSSv23 (IBM Corp., 2015). Mean and standard deviation were presented for characteristics of the study sample. Normal distribution of data was assessed by KolmogorovSmirnoff and Shapiro-Wilk tests, and examination of QQ plots, frequency histograms and standard errors of skewness and kurtosis. Variables not normally distributed were $\log$ transformed and parametric methods of analysis were then used. Three (3) datasets were used for statistical analysis; the total sample of participants $(n=70)$ to avoid the potential for detection bias, a VAS subgroup with a minimum level of pain as indicated by 2.0 or greater on the VAS scale $(n=42)$, and an ODI subgroup with a minimum level of disability as indicated by $10.0 \%$ or greater on the ODI questionnaire $(n=52)$. Pearson correlation coefficients were used to identify relationships between anthropometric and adiposity variables with self-reported pain and disability. Stepwise regression analyses were performed to explain relationships between anthropometric and adiposity variables with pain and disability, as well as determine the proportion of variance in pain and disability explained by such variables. Adjusted $R$ square values were reported for significant relationships. Predictor variables included in the regression analysis were determined by the results of the correlation analysis, where only variables found to be correlated with pain or disability were included in the regression models to reduce the potential effect of confounding variables. The variance inflation factor (VIF) was used to determine the effect of collinearity of prediction variables on regression analyses. A VIF $>5$ for any two variables was used to indicate collinearity, in which case the variable with the higher VIF was removed from the prediction model. Missing data were addressed through exclusion of the incomplete variable/s for a given participant from the analysis model. The study
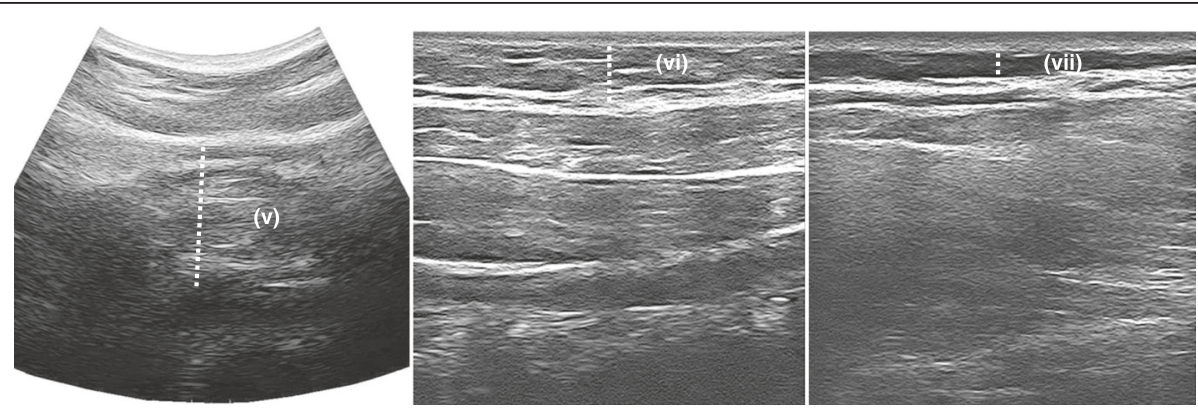

Fig. 2 Examples of intra-abdominal, supra-iliac and lumbar US measurements (v) maximum intra-abdominal adiposity (vi) maximum subcutaneous supra-iliac adiposity (vii) maximum subcutaneous lumbar adiposity 
Table 2 Ultrasound-derived adiposity variables

\begin{tabular}{|c|c|c|}
\hline Measure & Calculation & Definition \\
\hline$\overline{A-L}$ & $(\mathrm{MsAa}+\mathrm{MiA}) / \mathrm{MsL}$ & $\begin{array}{l}\text { Abdominal-to-lumbar adiposity ratio (total subcutaneous and visceral abdominal } \\
\text { adiposity thickness relative to lumbar adiposity thickness) }\end{array}$ \\
\hline S-M & $(\mathrm{MsAa}+\mathrm{MsSI}+\mathrm{MsL}) /$ weight & $\begin{array}{l}\text { Subcutaneous adiposity to mass ratio (total subcutaneous trunk adiposity thickness } \\
\text { relative to overall body mass) }\end{array}$ \\
\hline V-M & $(\mathrm{Mpp} A+\mathrm{MiA}) /$ weight & $\begin{array}{l}\text { Visceral adiposity to mass ratio (total visceral trunk adiposity thickness relative to overall } \\
\text { body mass) }\end{array}$ \\
\hline MAR-A & $\mathrm{MsAa} / \mathrm{MiA}$ & Maximal abdominal ratio A (ratio between subcutaneous and visceral abdominal adiposity) \\
\hline $\mathrm{A}-\mathrm{L} / \mathrm{BMI}$ & $[(\mathrm{MsAa}+\mathrm{MiA}) / \mathrm{MsL}] /[$ weight/(height $\times$ height $)]$ & $\begin{array}{l}\text { Abdominal-to-lumbar adiposity ratio to BMI (ratio between abdominal and lumbar } \\
\text { adiposity thickness relative to overall body mass index }\end{array}$ \\
\hline $\mathrm{A}-\mathrm{L} / \mathrm{WHR}$ & $\begin{array}{l}{[(M s A a+M i A) / M s L] /(\text { waist circumference/hip }} \\
\text { circumference })\end{array}$ & $\begin{array}{l}\text { Abdominal-to-lumbar adiposity ratio to WHR (ratio between abdominal and lumbar } \\
\text { adiposity thickness relative to the ratio between waist and hip circumferences) }\end{array}$ \\
\hline TC-TBA & $\begin{array}{l}(\mathrm{MsAa}+\mathrm{MsSI}+\mathrm{MsL}) / \text { total body adiposity } \\
\text { percentage }\end{array}$ & $\begin{array}{l}\text { Total circumference to total body adiposity ratio (total trunk circumference thickness } \\
\text { relative to total body adiposity percentage) }\end{array}$ \\
\hline
\end{tabular}

MppA maximum pre-peritoneal abdominal adiposity, MsAa maximum subcutaneous abdominal adiposity A, MiA maximum intra-abdominal adiposity, MsSI maximum subcutaneous supra-iliac adiposity, MsL maximum subcutaneous lumbar adiposity

size was arrived at with the use of post-hoc calculations of statistical power. Statistical significance was set at $p<0.05$.

\section{Results}

A total of $n=122$ individuals were screened for inclusion and $n=70$ cLBP individuals were eligible and chose to participate in the study. The characteristics of the study sample are summarised in Tables 3, 4 and 5. One (1) participant had missing data of the minimum subcutaneous lumbar adiposity measurement.

\section{Relationship between anthropometric and adiposity measures to pain and disability}

Correlations between anthropometric and adiposity measures with pain are shown in Table 6. There were no significant correlations observed between self-reported

Table 3 Demographic characteristics of the study sample $(n=70)$

\begin{tabular}{lc}
\hline Age (yrs) & $39.57 \pm 11.01$ \\
CLBP (yrs) & $9.84 \pm 8.60$ \\
Gender (M/F) & $30 \mathrm{M}, 40 \mathrm{~F}$ \\
Height (m) & $1.70 \pm 0.08$ \\
Weight (kg) & $79.66 \pm 17.44$ \\
BMl (kg/m $\left.{ }^{2}\right)$ & $27.49 \pm 5.63$ \\
WC (cm) & $87.72 \pm 14.68$ \\
HC (cm) & $104.94 \pm 10.03$ \\
WHR & $0.83 \pm 0.09$ \\
TBA & $29.99 \pm 10.87$ \\
ODI & $16.66 \pm 9.65$ \\
VAS & $2.38 \pm 1.78$ \\
\hline SD data mean \pm \\
CLBP chronic low back pain, BMI body mass index, WC waist circumference, HC \\
hip circumference, WHR waist-to-hip ratio, TBA total body adiposity percentage, \\
ODl oswestry disability index, VAS visual analogue scale
\end{tabular}

disability and anthropometric or adiposity variables in any of the analysis models. ODI was found to be correlated to VAS in the total sample $(r=0.264, p=0.028)$, but not in either of the subgroup analysis models.

Stepwise regression showed that $9.1 \%(p=0.007)$ of the variance in pain was explained by A-L alone in the total sample analysis $(n=70)$, which was increased to $15.7 \%(p=0.001)$ when ODI was added to the model. Results of the stepwise regression for the VAS subgroup indicated that $30.5 \%$ of the variance in pain could be explained by A-L/WHR $(p<0.001)$. Similar results were observed in the ODI subgroup regression analysis, as $24.7 \%$ of the variance in pain was explained by A-L/BMI $(p<0.001)$. No regression analysis was performed on self-reported disability on the basis of no significant correlations to anthropometric or adiposity variables in any of the analysis models. Post-hoc results revealed an achieved statistical power of $\beta=0.75$ for the variance in pain explained by $\mathrm{A}-\mathrm{L} / \mathrm{WHR}$.

Table 4 Absolute ultrasound measurements $(\mathrm{mm})$ of the study sample $(n=70)$

\begin{tabular}{ll}
\hline msA & $12.34 \pm 7.79$ \\
MppA & $13.36 \pm 4.53$ \\
MsAa & $20.19 \pm 9.69$ \\
MsAb & $19.60 \pm 9.90$ \\
MiA & $49.77 \pm 23.01$ \\
MsSI & $14.40 \pm 7.69$ \\
MsL & $8.36 \pm 6.90$
\end{tabular}

Data mean \pm SD

$m s A$ minimum subcutaneous abdominal adiposity, MppA maximum preperitoneal abdominal adiposity, MsAa maximum subcutaneous abdominal adiposity A, MsAb maximum subcutaneous abdominal adiposity B, MiA maximum intra-abdominal adiposity, MsSI maximum subcutaneous supra-iliac adiposity, MsL maximum subcutaneous lumbar adiposity 
Table 5 Relative ultrasound measurements and ratios of the study sample $(n=70)$

\begin{tabular}{ll}
\hline A-L & $12.42 \pm 9.12$ \\
S-M & $0.54 \pm 0.24$ \\
V-M & $0.78 \pm 0.20$ \\
MAR-A & $0.46 \pm 0.25$ \\
A-L/BMI & $0.47 \pm 0.38$ \\
A-L/WHR & $14.44 \pm 10.11$ \\
TC-TBA & $1.44 \pm 0.52$ \\
\hline
\end{tabular}

Data mean \pm SD

$A-L$ abdominal to lumbar adiposity ratio, $S-M$ subcutaneous adiposity to mass ratio, $V-M$ visceral adiposity to mass ratio, MAR-A maximum abdominal ratio $\mathrm{A}$, $A-L / B M I$ abdominal to lumbar adiposity ratio to $B M I, A-L / W H R$ abdominal to lumbar adiposity ratio to WHR, TC-TBA total circumference to total body adiposity ratio

\section{Discussion}

It was hypothesised that greater abdominal adiposity, particularly visceral, would be associated with increased self-reported pain and disability in cLBP individuals. This study's findings showed a relationship between anthropometric and adiposity measures to self-reported pain in CLBP, but not disability. More specifically, A-L relative to the size of the individual was the best predictor of self-reported pain.

The results of this study support previous suggestions that visceral adiposity may be more important than subcutaneous adiposity in the relationship to pain. For example, the overflow of adipocytes into excess visceral and ectopic stores may initiate a process of metabolic dysfunction [46] resulting from the disrupted equilibrium between energy intake and lipid oxidation [36]. Consequently, this overflow may promote the release of adipocyte-derived pro-inflammatory cytokines [47] contributing to insulin resistance and end-stage disease $[46,47]$, but also to hyperalgesia and central sensitisation

Table 6 Significant correlations between anthropometric and adiposity variables with self-reported pain

\begin{tabular}{llll}
\hline Analysis model & Variable & $r$ & $p$ \\
\hline Total sample $(n=70)$ & A-L & 0.323 & 0.007 \\
& A-L/WHR & 0.315 & 0.008 \\
& A-L/BMI & 0.303 & 0.011 \\
VAS subgroup $(n=42)$ & A-L & 0.566 & $<0.001$ \\
& A-L/WHR & 0.568 & $<0.001$ \\
& A-L/BMI & 0.546 & $<0.001$ \\
ODI subgroup $(n=52)$ & A-L & 0.493 & $<0.001$ \\
& A-L/WHR & 0.438 & 0.001 \\
& A-L/BMI & 0.5111 & $<0.001$ \\
& WHR & 0.287 & 0.039 \\
\hline
\end{tabular}

VAS visual analogue scale, $O D I$ oswestry disability index, $A-L$ abdominal to lumbar adiposity ratio, $A-L / W H R$ abdominal to lumbar adiposity ratio to WHR, $A-L / B M I$ abdominal to lumbar adiposity ratio to $B M I$ WHR, waist-to-hip ratio
[48]. In addition to metabolic dysfunction, there is growing evidence for the pathophysiological consequences on bone and skeletal muscle integrity and function from abnormal lipid accumulation [36]. The result may then be chronic low-grade systemic inflammation [46, 47] and therefore the persistence of a chronic pain state [48]. For example, increased levels of C-reactive protein, a sensitive acute-phase protein associated with body adiposity measures [49], has been linked to greater odds of reporting LBP symptoms, particularly in those measured as obese by BMI or WC [49]. It has been suggested that increased $\mathrm{C}$-reactive protein may be indicative of early signs of lowgrade chronic systemic inflammation [49]. Consequently, it may validate the implication of pro-inflammatory cytokines in the complex pathways of musculoskeletal pain [49] and further support the use of visceral adiposity measurements, such as US, in the research of cLBP and other chronic pain pathologies.

This study's findings may also support a theorised metabolic mediation in the adiposity-pain relationship [12]. Since pain was found to be significantly correlated with A-L relative to BMI or WHR, visceral adiposity relative to body size and shape may be an important consideration for future research. For example, the distribution of A-L may be just as important as the overall representation of body size and mass distribution. Therefore, it may be the accumulation of body mass coupled with greater levels of relative adiposity that puts an individual in an increased or more persistent cLBP state.

The moderate to strong correlations and prediction models between pain and A-L relative to WHR and BMI may advocate a possible physiological or biomechanical mediation between obesity and cLBP. For instance, WHR measures an individual's anatomical circumference of the waist compared to the hips to assess adiposity distribution [50] and the associated risk of deviating from optimal body morphology for physical health. In turn, BMI is a measure of overall body size as a relative association between height and weight [15], with an optimum balance to achieve the 'healthy' range. Consequently, coupling WHR and BMI with the A-L/pain relationship may further support a physiological or biomechanical mediation. It is reasonable to believe that the body can only manage a degree of anterior-toposterior load, but is also functionally limited by waistto-hip load and overall body load. For example, perhaps an individual with a high A-L, large WHR and elevated BMI may be in greater pain than someone with the same A-L but lower WHR and BMI. It may be the accumulation of the overall body mass and weight distribution including adiposity that acts as a pain catalyst, but is the A-L that is most instrumental in observable and measurable biomechanical changes. For instance, it is plausible 
that greater anterior mass may result in increased compensatory lordosis during normal daily posture, manifest by spinal hyperextension, and thereby excess abdominal adiposity may result in increased magnitude or repetition of compression loading, which is a known precursor for risk of intervertebral disc injury [26]. Moreover, previous research suggests that both vertebral joint compression and postural deviation may impact upon shear injury potential [51]. Irrespective of these yet unconfirmed inferences, it is known that obesity and cLBP are linked [3-13] and that simplistic measurements like BMI are unrelated to CLBP [52] and lack the sensitivity to detect excessive adiposity in non-obese individuals [36]. Therefore, future research may need to explore more comprehensive measurements such as A-L to further quantify and explain the adiposity-cLBP relationship.

The hypothesis that greater abdominal adiposity would be associated with increased disability was not supported, as no correlations were found between anthropometric and adiposity variables with disability. This finding was not supported or refuted by previous research, since no other studies to the authors' knowledge have examined the relationship between adiposity and disability associated with cLBP. An earlier study reporting a relationship between adiposity and disability associated with LBP [12] was not specific to CLBP and assessed adiposity and disability using different methods to those used in this study. Therefore, further research may be necessary to confirm that adiposity and disability are unrelated in CLBP.

The novelty of this research lends itself to potential constraints, such as the use of absolute and relative adiposity ratios not previously studied. The removal of variables to eliminate collinearity during statistical analysis may have excluded potentially relevant variables from the prediction models. However, any variables removed were those with the least impact on the prediction models. Correlation analysis between each variable with pain and disability also ensured all relevant relationships between variables were explored. It may be irrelevant which A-L variables were left in the regression analyses, since all A-L variables were found to have strong correlations to pain. The use of WHR instead of WC may be a limitation since adipose tissue deposits in the abdominal versus gluteofemoral region may have different biological mechanisms and therefore altered health risk implications [50]. For this reason, future studies into the A-L/cLBP relationship may benefit more from the use of WC instead of WHR. The selection of VAS and ODI cutoff values may have excluded potentially relevant data, but since the majority of existing research explored the minimum level of clinically meaningful change over time no previous consensus on normative scores for minimal pain or disability levels in cLBP was found.
Therefore, values were set from collaborative evidence of minimal important change values in VAS ranging from 1.5-2.0 [53] and a normative score of 10.19 for ODI of 'normal' populations [42], which was deemed appropriate based on available evidence. The study results can only be generalised to adult cLBP populations.

\section{Conclusions}

The results of this study demonstrated significant relationships between abdominal adiposity and cLBP. A-L combined with increased WHR and BMI was a predictor of pain variance. Therefore, an individual's adiposity distribution relative to their body or trunk mass may be of greater importance in the cLBP-obesity relationship than single measurements alone. These findings support the use of US-based methodologies for future cLBP research. Until the mechanisms responsible for the adiposity-cLBP relationship are better understood, attempts to manipulate it through pain or adiposity reduction treatment may be of little benefit. For this reason, additional research into possible physiological, metabolic and biomechanical mediators between adiposity distribution and pain manifestation in cLBP is warranted.

\section{Abbreviations \\ A-L, abdominal to lumbar adiposity ratio; A-L/WHR, abdominal to lumbar adiposity ratio relative to waist-to-hip ratio; A-L/BMl, abdominal to lumbar adiposity ratio relative to body mass index; $B I A$, bioelectrical impedance analysis; BMI, body mass index; CLBP, chronic low back pain; LBP, Low back pain; ODI, \\ Oswestry Disability Index; US, ultrasound; VAS, visual analogue scale; VIF, variance inflation factor; WC, waist circumference; WHR, waist-to-hip ratio}

\section{Acknowledgements}

The authors wish to acknowledge all research participants involved in the study. This study was performed within the Sport and Exercise Science department of the School of Science and Health, Western Sydney University, Australia.

\section{Funding}

The authors wish to acknowledge the Commonwealth Government of Australia for the provision of the Australian Postgraduate Award (APA) to the lead author of this manuscript and subsequent funding of this research.

\section{Availability of data and material}

The datasets generated during and/or analysed during the current study are not publicly available due to sufficient information to support the conclusions of this article being included and presented within the text of the manuscript, but are available from the corresponding author on reasonable request.

\section{Authors' contributions}

$\mathrm{CB}$ contributed to the design of the study, participant recruitment, acquisition of data, statistical data analysis and drafting of the manuscript. JCS contributed to the draft of the manuscript. PWMM contributed to the design of the study, statistical analysis and drafting of the manuscript. All authors read and approved the final manuscript.

\section{Competing interests}

The author(s) declare that they have no competing interests.

Consent for publication

Not applicable. 


\section{Ethics approval and consent to participate}

This study was approved for human subjects' research by the Human Research Ethics Committee of Western Sydney University on the basis of the Declaration of Helsinki (H9540/H10512). Written informed consent was provided by all participants.

\section{Author details}

'School of Science and Health, Western Sydney University, Locked Bag 1797Campbelltown Campus, Sydney, NSW 2751, Australia. ${ }^{2}$ School of Science and Health, Western Sydney University, Campbelltown Campus, Sydney, Australia.

Received: 11 May 2016 Accepted: 22 July 2016

\section{Published online: 02 August 2016}

\section{References}

1. Walker BF, Muller R, Grant WD. Low back pain in Australian adults: the economic burden. Asia Pac J Public Health. 2003;15:79-87.

2. Murray CJ, Lopez AD. Measuring the global burden of disease. N Engl J Med. 2013:369:448-57

3. Bener A, Alwash R, Gaber T, et al. Obesity and low back pain. Coll Antropol. 2003;27:95-104.

4. Deyo RA, Bass JE. Lifestyle and low-back pain. The influence of smoking and obesity. Spine. 1989;14:501-6.

5. Han TS, Schouten JS, Lean ME, et al. The prevalence of low back pain and associations with body fatness, fat distribution and height. Int J Obes Relat Metab Disord. 1997;21:600-7.

6. Heuch I, Hagen K, Nygaard O, et al. The impact of body mass index on the prevalence of low back pain: the HUNT study. Spine. 2010;35:764-8.

7. Leboeuf-Yde C. Body weight and low back pain. A systematic literature review of 56 journal articles reporting on 65 epidemiologic studies. Spine. 2000;25:226-37.

8. Leboeuf Yde C, Kyvik KO, Bruun NH. Low back pain and lifestyle. Part IIObesity. Information from a population-based sample of 29,424 twin subjects. Spine. 1999:24:779-83. discussion 783-774.

9. Mirtz TA, Greene L. Is obesity a risk factor for low back pain? An example of using the evidence to answer a clinical question. Chiropr Osteopat. 2005:13:2

10. Nilsen Tl, Holtermann A, Mork PJ. Physical exercise, body mass index, and risk of chronic pain in the low back and neck/shoulders: longitudinal data from the Nord-Trondelag Health Study. Am J Epidemiol. 2011;174:267-73.

11. Shiri R, Solovieva S, Husgafvel-Pursiainen $K$, et al. The association between obesity and the prevalence of low back pain in young adults: The Cardiovascular Risk in Young Finns Study. Am J Epidemiol. 2008;167:1110-9.

12. Urquhart DM, Berry P, Wluka AE, et al. 2011 Young Investigator Award winner: Increased fat mass is associated with high levels of low back pain intensity and disability. Spine. 2011;36:1320-5.

13. Wright $L$, Schur E, Noonan C, et al. Chronic pain, overweight, and obesity: findings from a community-based twin registry. J Pain. 2010;11:628-35.

14. Mangwani J, Giles C, Mullins $M$, et al. Obesity and recovery from low back pain: a prospective study to investigate the effect of body mass index on recovery from low back pain. Ann R Coll Surg Engl. 2010;92:23-6.

15. WHO. Obesity: preventing and managing the global epidemic. Report of a WHO consultation. World Health Organ Tech Rep Ser. 2000:894:i-xii, 1-253

16. Bazzocchi A, Filonzi G, Ponti F, et al. Accuracy, reproducibility and repeatability of ultrasonography in the assessment of abdominal adiposity. Acad Radiol. 2011;18:1133-43

17. De Lucia RE, Sleigh A, Finucane FM, et al. Ultrasound measurements of visceral and subcutaneous abdominal thickness to predict abdominal adiposity among older men and women. Obesity. 2010;18:625-31.

18. Eston R, Evans R, Fu F. Estimation of body composition in Chinese and British men by ultrasonographic assessment of segmental adipose tissue volume. Br J Sports Med. 1994;28:9-13.

19. Fanelli MT, Kuczmarski RJ. Ultrasound as an approach to assessing body composition. Am J Clin Nutr. 1984;39:703-9.

20. Holzhauer S, Zwijsen RM, Jaddoe WW, et al. Sonographic assessment of abdominal fat distribution in infancy. Eur J Epidemiol. 2009:24:521-9.

21. Vlachos IS, Hatziioannou A, Perelas A, et al. Sonographic assessment of regional adiposity. AJR Am J Roentgenol. 2007:189:1545-53.

22. Deschenes $D$, Couture $P$, Dupont $P$, et al. Subdivision of the subcutaneous adipose tissue compartment and lipid-lipoprotein levels in women. Obes Res. 2003;11:469-76.
23. Fox CS, Massaro JM, Hoffmann U, et al. Abdominal visceral and subcutaneous adipose tissue compartments: association with metabolic risk factors in the Framingham Heart Study. Circulation. 2007;116:39-48.

24. Shuster A, Patlas M, Pinthus JH, et al. The clinical importance of visceral adiposity: a critical review of methods for visceral adipose tissue analysis. $\mathrm{Br}$ I Radiol. 2012;85:1-10.

25. Ibrahim MM. Subcutaneous and visceral adipose tissue: structural and functional differences. Obes Rev. 2010;11:11-8.

26. Callaghan JP, McGill SM. Intervertebral disc herniation: studies on a porcine model exposed to highly repetitive flexion/extension motion with compressive force. Clin Biomech. 2001;16:28-37.

27. Boneva-Asiova Z, Boyanov MA. Body composition analysis by leg-to-leg bioelectrical impedance and dual-energy X-ray absorptiometry in nonobese and obese individuals. Diabetes Obes Metab. 2008;10:1012-8.

28. Chouinard LE, Schoeller DA, Watras AC, et al. Bioelectrical impedance vs. four-compartment model to assess body fat change in overweight adults. Obesity. 2007;15:85-92.

29. Kyle UG, Bosaeus I, De Lorenzo AD, et al. Bioelectrical impedance analysispart I: review of principles and methods. Clin Nutr. 2004:23:1226-43.

30. Medrano G, Eitner F, Walter M, et al. Model-based correction of the influence of body position on continuous segmental and hand-to-foot bioimpedance measurements. Med Biol Eng Comput. 2010;48:531-41.

31. Pateyjohns IR, Brinkworth GD, Buckley JD, et al. Comparison of three bioelectrical impedance methods with DXA in overweight and obese men. Obesity. 2006;14:2064-70.

32. Ritchie JD, Miller CK, Smiciklas-Wright H. Tanita foot-to-foot bioelectrical impedance analysis system validated in older adults. J Am Diet Assoc. 2005:105:1617-9.

33. Rush EC, Crowley J, Freitas IF, et al. Validity of hand-to-foot measurement of bioimpedance: standing compared with lying position. Obesity. 2006;14:252-7.

34. Thomson R, Brinkworth GD, Buckley JD, et al. Good agreement between bioelectrical impedance and dual-energy $\mathrm{X}$-ray absorptiometry for estimating changes in body composition during weight loss in overweight young women. Clin Nutr. 2007;26:771-7.

35. Bigaard J, Frederiksen K, Tjonneland A, et al. Waist and hip circumferences and all-cause mortality: usefulness of the waist-to-hip ratio? Int J Obes Relat Metab Disord. 2004;28:741-7.

36. Peterson MD, Zhang P, Haapala HJ, et al. Greater Adipose Tissue Distribution and Diminished Spinal Musculoskeletal Density in Adults With Cerebral Palsy. Arch Phys Med Rehabil. 2015;96:1828-33.

37. Marshall P, Murphy B. Delayed abdominal muscle onsets and self-report measures of pain and disability in chronic low back pain. J Electromyog Kinesiol. 2010:20:833-9.

38. Marshall PW, Murphy BA. Evaluation of functional and neuromuscular changes after exercise rehabilitation for low back pain using a Swiss ball: a pilot study. J Manipulative Physiol Ther. 2006;29:550-60.

39. Marshall PW, Murphy BA. Muscle activation changes after exercise rehabilitation for chronic low back pain. Arch Phys Med Rehabil. 2008;89:1305-13.

40. Bijur PE, Silver W, Gallagher EJ. Reliability of the visual analog scale for measurement of acute pain. Acad Emerg Med. 2001;8:1153-7.

41. Chapman JR, Norvell DC, Hermsmeyer JT, et al. Evaluating common outcomes for measuring treatment success for chronic low back pain Spine. 2011:36:554-68.

42. Fairbank JC, Pynsent PB. The Oswestry Disability Index. Spine. 2000;25:2940-52. discussion 2952

43. Hawker GA, Mian S, Kendzerska T, et al. Measures of adult pain: Visual Analog Scale for Pain (VAS Pain), Numeric Rating Scale for Pain (NRS Pain), McGill Pain Questionnaire (MPQ), Short-Form McGill Pain Questionnaire (SF-MPQ), Chronic Pain Grade Scale (CPGS), Short Form-36 Bodily Pain Scale (SF-36 BPS), and Measure of Intermittent and Constant Osteoarthritis Pain (ICOAP). Arthritis Care Res (Hoboken). 2011;63 Suppl 11:S240-52.

44. Price DD, McGrath PA, Rafii A, et al. The validation of visual analogue scales as ratio scale measures for chronic and experimental pain. Pain. 1983;17:45-56.

45. Roland M, Fairbank J. The Roland-Morris Disability Questionnaire and the Oswestry Disability Questionnaire. Spine. 2000;25:3115-24.

46. Egger G, Dixon J. Non-nutrient causes of low-grade, systemic inflammation: Support for a 'canary in the mineshaft' view of obesity in chronic disease. Obes Rev. 2011:12:339-45.

47. Gleeson M, Bishop NC, Stensel DJ, et al. The anti-inflammatory effects of exercise: mechanisms and implications for the prevention and treatment of disease. Nat Rev Immunol. 2011;11:607-15. 
48. Farquhar-Smith WP. Anatomy, physiology and pharmacology of pain. Anaesth Intensive Care. 2008;9:3-7.

49. Briggs MS, Givens DL, Schmitt $L C$, et al. Relations of $C$-reactive protein and obesity to the prevalence and the odds of reporting low back pain. Arch Phys Med Rehabil. 2013;94:745-52.

50. Bigaard J, Thomsen BL, Tjonneland A, et al. Does waist circumference alone explain obesity-related health risk? Am J Clin Nutr. 2004;80:790-1. author reply $791-792$

51. Howarth SJ, Callaghan JP. Compressive force magnitude and intervertebral joint flexion/extension angle influence shear failure force magnitude in the porcine cervical spine. J Biomech. 2012;45:484-90.

52. Brooks C, Siegler JC, Cheema BS, et al. No Relationship Between Body Mass Index and Changes in Pain and Disability After Exercise Rehabilitation for Patients With Mild to Moderate Chronic Low Back Pain. Spine. 2013;38:1-6.

53. Ostelo RW, Deyo RA, Stratford P, et al. Interpreting change scores for pain and functional status in low back pain: towards international consensus regarding minimal important change. Spine. 2008;33:90-4.

Submit your next manuscript to BioMed Central and we will help you at every step:

- We accept pre-submission inquiries

- Our selector tool helps you to find the most relevant journal

- We provide round the clock customer support

- Convenient online submission

- Thorough peer review

- Inclusion in PubMed and all major indexing services

- Maximum visibility for your research

Submit your manuscript at www.biomedcentral.com/submit
) Biomed Central 